Bull. Fac. Agric., Cairo Univ., 58( 2007): 1-10.

\title{
EFFECT OF PLANT DISTRIBUTION PATTERN AND NITROGEN FERTILIZATION ON YIELD AND PHOTOSYNTHATES PARTITIONING OF SAFFLOWER
}

(Received:14.8.2006)

\author{
By \\ S.A.I. Ghanem and S. M.I. Ash-Shormillesy \\ Department of Agronomy, Faculty of Agriculture, Zagazig University
}

\begin{abstract}
A field study was excuted during 2002/2003 and 2003/2004 seasons at Khattara Project Farm (newly reclaimed sandy soil), Fac. of Agric., Zagazig Univ., Egypt to test the effect of four plant distribution patterns, being $\mathrm{P}_{1}(60 \times 20 \mathrm{~cm}, 1$ plant $/$ hill, 1 side of the ridge $=35000$ plant $/$ fed. $), \mathrm{P}_{2}(60 \times 30 \mathrm{~cm}, 1$ plant /hill, 2 sides of the ridge $=46666$ plant/fed. $), \mathrm{P}_{3}(60 \times 20 \mathrm{~cm}, 2$ plants $/$ hill, 1 side of the ridge $=$ 70000 plant $/ \mathrm{fed})$ and $\mathrm{P}_{4}(60 \times 30 \mathrm{~cm}, 2$ plants $/$ hill, 2 sides of the ridge $=93333$ plant $/ \mathrm{fed}$. $)$ as well as $\mathrm{N}$ fertilization levels (without $\mathrm{N}$ application, 40 and $80 \mathrm{~kg} \mathrm{~N} / \mathrm{fed}$.) on the yield and photosynthate partitioning respects of both Giza 1 and Demo 112 safflower cultivars.

The two safflower cultivars showed significant differences with respect most of the studied traits, where Giza $1 \mathrm{cv}$ gave greater mean values respecting RPP of straw yield/plant than Demo 112 which was superior to the former $\mathrm{cv}$ in each of head dry weight /plant, relative photosynthetic potential (RPP) of seed yield/plant, biomass/plant, migration coefficient (MC) and the final yields/plant from straw, seed and biomass. On the other hand, no significant cultivar variance was seen regarding their Leaf area index (LAI). Such cultivar behaviour was observed in both seasons.

The four plant distribution patterns gave remarked differences among them as for all the studied respects, since the planting pattern of $60 \times 20 \mathrm{~cm}, 1$ plant/hill, 1 side of the ridge $(35000 \mathrm{plant} / \mathrm{fed}$.) reflected the greatest records in each of : head dry weight/plant and the final yield / plant from straw, seed and biomass as well as their relative photosynthetic potentials, when compared with the other three patterns used. On the other hand, the safflower plants established by $60 \times 30 \mathrm{~cm} \times$ two plants/hill, two sides of the ridge (93333 plant stand density/fed.) possessed greater records regarding : LAI and (MC) followed in ranking by $\mathrm{P}_{3}, \mathrm{P}_{2}$ and $\mathrm{P}_{1}$ of plant densities with 70000 , 46666 and 93333 thousand plant/fed., orderly. Such planting pattern behaviour was distinguished in both individual seasons.

In addition, the three $\mathrm{N}$ levels secured greater changes in their photosynthate partitioning parameters in both trials and over them, where the $80 \mathrm{~kg} \mathrm{~N}$ dose achieved the greatest averages in each of : LAI, head dry weight/plant, MC and the final yields/plant from straw, seed and biomass as well as their relative photothenthetic potentials when compared with the $40 \mathrm{~kg} \mathrm{~N}$ level and the check treatment.

The 3 factors tested interacted strongly as for most of the studied traits, but the best results were seen due to fertilizing imported $12 \mathrm{cv}$ with $80 \mathrm{~kg} \mathrm{~N}$ level and by using either $\mathrm{P}_{1}$ pattern $(60 \mathrm{x} 20 \mathrm{~cm}, 1$ plant/hill, 1 side of the ridge with plant stand density of 35000 plant/fed) as for the final straw, seed, biomass and their RPP or $\mathrm{P}_{4}$ system $(60 \times 20 \mathrm{~cm}$, two plants/hill, two sides of the ridge $=93333$ plant/fed.) respecting LAI and migration coefficient.

In brief, the safflower plants of Demo 112 cultivar proved to have a better canopy structure and can produce greater photosynthate partitioning respects and the good yields/plant when were probably established by the even distribution pattern of 35000 plant/fed. and using the $80 \mathrm{~kg} \mathrm{~N}$ level especially in the newly reclaimed sandy soils of poor fertility as those found in Khattara Project Farm, Zagazig location, Sharkia Governorate, Egypt.
\end{abstract}

Key words : fertilization, photosynthates, safflower, yield.

\section{INTRODUCTION}

Safflower (Carthamus tinctorious L.) is considered as one of the most important oil crops in the world due to its numerous uses of both flower petals and edible oil, as well as the special advantage of its plants to grow well in barren soils. In Egypt, the Government is pressing hard to increase safflower production, especially in newly 
reclaimed sandy lands of little rainfall such as Toshky and North Sinai locations, to bridge the nutritious gab found between the decreased production of edible oil and the increased consumption caused by over population. So, to solve such a problem, the appropriate cultural practices should be applied such as growing the highly yielding cvs either local or introduced under modern cultural techniques such as even distributing planting patterns and suitable $\mathrm{N}$ fertilization levels, which could help in increasing the photosynthesis capacity of safflower plants resulting in enhancing of more metabolites inside plant organs and maximizing the consequent yield. Three of the most important factors affecting yield and dry matter partition are promising cultivars, plant density and $\mathrm{N}$ fertilization levels.

Many workers evaluated the safflower cultivar behaviours in most agronomic characters, of them: Khalil (1988) reported significant cultivar differences respecting leaf area (LA), LAI, dry weight (DW)/plant (at different growth stages), straw and seed yields/plant and seed yield efficiency, where the Demo 112 cultivar exceeded both Imported 63 and Giza 1 in such mentioned traits. Also, similar findings were documented by El-Wakil and Darweesh (1993) Likewise, Refaat et al. (1994) indicated that the Portogalian safflower $\mathrm{cv}$ was superior to the Egyptian, Sweiss and Romanian ones as for head weight/plant, seed weight/head and seed yield/plant.

Regarding the plant distribution pattern effect, Kamel et al., (1982) cited that the safflower dry weight/plant, LA and relative growth rate (RGR) recorded at different growth stages were markedly increased due to $30 \mathrm{~cm}$ hill spacing when compared with both 10 and $20 \mathrm{~cm}$ spaces. In contrast, LAI showed a significant excess in favour of the narrowest hill space of $10 \mathrm{~cm}$ apart. Besides, Khalil (1988) showed that safflower plant height and LAI were of greater values due to increasing plant density from 40 to 56 and 93 thousand plant/fad (Shweikh, 1988 recorded similar views in this regard). In addition, El-Hariri and Ahmed (1993) stated that the 70000 plant stand/fad exceeded both 35 and 105000 plant/fad respecting plant height, straw, seed and biological yields per plant or/fed. On the contrary, the number of branches and heads/plant, head dry weight/plant, migration coefficient (MC), seed index and relative photosynthetic potential (RPP) from straw, seed and biomass/plant were significantly decreased by raising plant density more than 35000 plant/fed. Together with, Nawar
(2002) established safflower plants under 15, 20 and $25 \mathrm{~cm}$ between hills and cited that stem diamer, number of branches and heads/plant and seed yield/plant were markedly raised by using the widest hill spacing of $25 \mathrm{~cm}$ apart. But, the reverse hold true as for seed yield/fed.

Respecting $\mathrm{N}$ fertilization effect, Khalil (1988) mentioned that safflower dry weight/plant, LA/plant and LAI showed marked increases with $90 \mathrm{~kg} \mathrm{~N}$ level when compared with 30 and 60 $\mathrm{kg} / \mathrm{fed}$. Similar trend was documented by Afifi (1991). Likewise, El-Hariri and Ahmed (1993) mentioned that plant height, head dry weight/plant, seed index, straw yield/plant and its RPP were significantly increased in response to the application of $60 \mathrm{~kg} \mathrm{~N} / \mathrm{fad}$ when compared with 20 and $40 \mathrm{~kg} \mathrm{~N}$ ones. While, the number of heads/plant, migration coefficient and RPP of both seed and biological yields/plant were statistically decreased by increasing $\mathrm{N}$ fertilizer level than 40 $\mathrm{kg} \mathrm{N} /$ fed. Moreover, Badawi et al. (1996) in their study on safflower concluded that the $75 \mathrm{~kg} \mathrm{~N}$ level increased meaningly the number of heads/plant, seed number/head, seed index and subsequently seed yield/plant relative to 45 and 60 $\mathrm{kg} \mathrm{N}$ levels and the check treatment. Whithal, Nawar (2002) working on safflower cv Giza 1 demonstrated that using $80 \mathrm{~kg} \mathrm{~N}$ level increased significantly stem diameter, heads/plant, seed index and seed yield either / plant or /fed when compared with both 40 and $60 \mathrm{~kg} \mathrm{~N}$ levels.

Accordingly, this work aimed to investigate the effect of plant distribution patterns and $\mathrm{N}$ fertilization levels on the yield and photosynthate partitioning respects of both Giza 1 and Demo 112 safflower cultivars grown under newly reclaimed sandy soil conditions.

\section{MATERIALS AND METHODS}

The present trials conducted herein were initiated during the two successive seasons of 2002/2003 and 2003/2004 at Khattara Project Farm, Fac. of Agric., Zagazig Univ., Sharkia Governorate, Egypt. The main objective of this work was to examine the effect of planting patterns and $\mathrm{N}$ fertilization levels on the yield and photosynthates partitioning respects of the two safflower cultivars. The soil of the trials was sandy in texture with $\mathrm{pH}$ of $7.5,0.20 \%$ organic matter and having 7.50, 12.1 and $60.5 \mathrm{ppm}$ available $\mathrm{N}, \mathrm{P}$ and $\mathrm{K}$, respectively (averages of the two seasons for the upper $25 \mathrm{~cm}$ of the soil).

\subsection{Factors studied}

\subsubsection{Cultivars, $\mathbf{V}$}

Two safflower cultivars were used as following: 
a- Giza 1, a local cultivar.

b- Demo 112 (an introduced cultivar).

The source of seeds of both cultivars was Oil Crops Section, ARC, Giza, Egypt.

\subsubsection{Planting pattern, $P$}

The planting pattern included : ridge width, hill spacing and the number of plants/hill.

The following plant distribution patterns were : $\mathrm{P}_{1}=60 \times 20 \mathrm{~cm}, 1$ plant/hill, 1 side of the ridge (35.000 plant/fed).

$\mathrm{P}_{2}=60 \times 30 \mathrm{~cm}, 1$ plant/hill, 2 sides of the ridge (46.666 plant/fed).

$\mathrm{P}_{3}=60 \times 20 \mathrm{~cm}, 2$ plant/hill, 1 side of the ridge (70.000 plant/fed).

$\mathrm{P}_{4}=60 \times 30 \mathrm{~cm}, 2$ plants $/$ hill, 2 sides of the ridge (93.333 plant/fed).

\subsubsection{Nitrogen fertilization, $N$}

The following $\mathrm{N}$ levels were used :

a- Check, without $\mathrm{N}$ application.

b- $40 \mathrm{~kg} \mathrm{~N} /$ fed.

c- $80 \mathrm{~kg} \mathrm{~N} / \mathrm{fed}$.

\subsection{Layout of the field trials}

The design of each experiment was split-split plot with three replicates. The two cultivars were the same as in the main plot, the four planting patterns were the same in the sub-plots and the 3 $\mathrm{N}$ levels were also the same in the sub-sub plots. Each sub-sub plot consisted of 6 ridges, $60 \mathrm{~cm}$ apart and $4 \mathrm{~m}$ long comprising an area of $14.4 \mathrm{~m}^{2}$. Any of the experimental unit was surrounded by ditches of $1.2 \mathrm{~m}$ width to avoid the lateral movement of the irrigation water to the adjacent plots. In each experimental unit, the 2 outer ridges were left as a border, whereas the 4 inner ridges of $9.6 \mathrm{~m}^{2}$ were used for the determination of the studied characters.

\subsection{Cultural practices}

The proceeding crop was sudan grass in both trials. The experimental fields were well prepared through 3 ploughings and levellings. Sprinkler irrigation system was followed in this study. Both phosphorus and potassium fertilizers were added fully prior to planting at the rates of $31.0 \mathrm{~kg} \mathrm{P}_{2} \mathrm{O}_{5}$ and $50 \mathrm{~kg} \mathrm{~K} \mathrm{~K}_{2} \mathrm{O} / \mathrm{fed}$. in the form of calcium super phosphate $\left(15.5 \% \quad \mathrm{P}_{2} \mathrm{O}_{5}\right)$ and potassium sulphate (48-52\% $\mathrm{K}_{2} \mathrm{O}$ ), orderly. The seeds of both Giza 1 and Demo 112 cultivars were mixed well with the recommended fungicide to control seed and seedling diseases. Seeding rate used for both cvs was $12 \mathrm{~kg} / \mathrm{fed}$. The seeds were planted by using the Afir method in hills of 20 or $30 \mathrm{~cm}$ apart on one or 2 sides of the ridge as per treatment. Planting was done on 8 November in both seasons. After a complete emergence, the seedlings in each hill were thinned to one or two plants/hill as per treatment tested. Nitrogen fertilizer levels of 40 or $80 \mathrm{~kg} \mathrm{~N} / \mathrm{fad}$ were added in the form of urea fertilizer $(46.5 \% \mathrm{~N})$ at four equal doses after thinning and by interval of 15 days. All other practices were manually adopted on proper time and as usually applied in safflower production. At last, harvesting was done on June $15^{\text {th }}$ in both seasons.

\subsection{Characters studied}

The following photosynthate partitioning parameters (respects) were recorded on ten individual plants:

1- Leaf area index (LAI) : recorded at 120 days of age.

2- Head dry weight/plant (gm).

3- Relative photosynthetic potential of straw yield/plant $\left(\mathrm{RPP}_{\text {straw }}\right)$.

Straw yield/plant (gm)

$=-\mathrm{gm} / \mathrm{LAI}$

LAI at 120 days-old

4- Relative photosynthetic potential of seed yield/plant $\left(\mathrm{RPP}_{\text {seed }}\right)$.

Seed yield/plant (gm)

$$
=-\mathrm{gm} / \mathrm{LAI}
$$

LAI at 120 days-old

5- Relative photosynthetic potential of biological yield/plant $\left(\mathrm{RPP}_{\text {boil }}\right)$

$=\mathrm{RPP}$ of both straw and seed yields/plant, $\mathrm{gm} / \mathrm{LAI}$

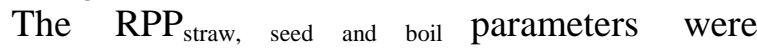
computed by following the procedure described by Vidovic and Pokorny (1971).

6- Migration coefficient (MC)

The migration coefficient of biomass/plant in gm was estimated using the manner outlined by McGraw (1977), as

Head dry weight $(\mathrm{gm}) / \mathrm{plant}$ at harvest

$\mathrm{MC}=\frac{}{\text { Biological yield/plant (gm), biomass }}$

7- Straw yield/plant (gm).

8- Seed yield/plant (gm).

9- Biological yield/plant (gm), biomass output.

\subsection{Statistical analysis}

The collected data of individual seasons were statistically analyzed by following the split-split plot system procedure as documented by Das and Giri (1986). In addition, the combined analysis of variance was also computed for the results of the two seasons, after establishing by Barlett's homogenity test, where the error variance of the individual years was homogeneous. The significant differences among the treatment means were judged with the help of Duncan's multiple range test (Duncan, 1955). In the interaction Tables recorded on the pooled data only, capital 
and small letters were used to compare both row and column averages, orderly. $*, * *$ and N.S are symbols in all listed Tables to verify the significant differences among treatment means at 5 and $1 \%$ levels of probability and insignificant differences, successively.

\section{RESULTS AND DISCUSSION \\ 3.1. Cultivar behavior}

The two safflower cultivars varied markedly as for such recorded characters in Tables 1,2 and 3, where Giza $1 \mathrm{cv}$ surpassed Demo 112 in relative photosynthetic potential of straw yield/plant $\left(\mathrm{RPP}_{\text {straw }}\right)$. On the contrary, the latter cultivar possessed greater mean records than the former in each of : head dry weight/plant, $\mathrm{RPP}_{\text {seed }}$,

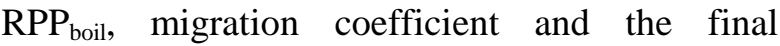
yields/plant from straw, seed and biomass. On the other hand, the difference between the two cultivars as for their LAI values did not reach the significancy level. Such cultivar behaviour was distinguished in both seasons (Tables 1, 2 and 3, orderly).

In general, the superiority of Demo 112 on its counterpart Giza 1 in these characters may be attributed inherently to the greater ability of such $\mathrm{cv}$ in synthesizing more assimilates that partitioned to the final economical yields of safflower plants and the consequent dry matter accumulation parameters discussed herein. Similar safflower cultivar differences were expressed by : Khalil (1988), El-Wakil and Darweesh (1993) and Refaat et al. (1994).

\subsection{Planting pattern effect}

The four planting patterns exhibited significant changes in all the studied characters recorded in Tables 1,2 and 3 . The $P_{1}$ pattern of $35000 \mathrm{plant} / \mathrm{fad}$ gave the greatest mean values in head dry weight/plant, the final yield/plant from straw, seed and biomass as well as their RPP, followed in order by $\mathrm{P}_{2}, \mathrm{P}_{3}$ and $\mathrm{P}_{4}$, successively. On the other hand, the dense plant distribution pattern of 93333 plant/fed. $(60$ x $20 \mathrm{~cm}, 2$ plants/hill and 2 sides of the ridge possessed high averages in LAI and migration coefficient. Such planting pattern trend was clearly valid in both trials and across them (Tables 1, 2 and 3, orderly).

It could be seen from the results recorded herein that, the better use of edaphic and aboveground environmental resources by the plants grown under the dense distribution pattern $\left(\mathrm{P}_{4}\right)$ may be completely attributed to such planting pattern excellence assembled herein as greater LAI and MC values. On the other hand, the superiority of light plant distribution pattern of 35000 plant/fad in most characters studied, being head dry weight/plant and the final yield/plant as well as their RPP might be explained by the most suitable distribution of safflower plants over the soil surface which resulted in a more effective use of light and other growth factors existed in the surrounding media which reflected their positive effect in raising the metabolites partitioned to the storage centres of safflower plants and raising the sequent yields. Analogous findings were documented by : Kamel et al. (1982), Khalil (1988), Shweikh (1988), El-Hariri and Ahmed (1993) and Nawar (2002).

\subsection{Nitrogen fertilization effect}

Significant variations between $\mathrm{N}$ levels were observed as for the discussed characters, the $80 \mathrm{~kg}$ $\mathrm{N}$ level secured the greatest mean values regarding LAI, head dry weight, RPP of straw, seed and biomass, MC and the final yield/plant from straw, seed and biomass followed by the $40 \mathrm{~kg} \mathrm{~N}$ level and the no- $\mathrm{N}$ fertilization treatment. This phenomenon was completely true in individual seasons and in their pooled data as well (Tables 1, 2 and 3). In poorly fertile soil like the one used in establishing these trials, the $80 \mathrm{~kg} \mathrm{~N}$ level was necessary in fertilization of safflower plants to increase the photosynthesis process and the consequent assimilates partitioned to the economic parts of safflower plants, which account much for increasing the final yields and the other photosynthate parameters. In other words $\mathrm{N}$ fertilization of safflower plants is completely required especially in newly sandy soils to enhance plant growth and to improve the transportation of more photosynthetic substances from the source to the sink during the vital synthetic processes. The effective role of $\mathrm{N}$ in raising safflower yield/plant and the other photosynthate respects is documented by other works, among them : Khalil (1988), Afifi (1991), El-Hariri and Ahmed (1993), Badawi et al. (1996) and Nawar (2002).

\subsection{Interaction effect}

The three factors under study interacted positively with each other with respects the dry matter partitioning parameters as follows : the $\mathrm{V} \mathrm{x}$ $\mathrm{P}$ interactions were significantly observed in each of $\mathrm{RPP}_{\text {straw, seed and biol. }}, \mathrm{MC}$ and the yield/plant from straw, seed and biomass (Table 4). The results show that Giza 1 plants under 35.000 plant/fed $\left(\mathrm{P}_{1}\right)$ gave high mean values as for $\mathrm{RPP}_{\text {straw. }}$. Also, the plants of Demo 112 cultivar grown under $\mathrm{P}_{1}$ pattern (35.000 plant/fed) possessed high records 


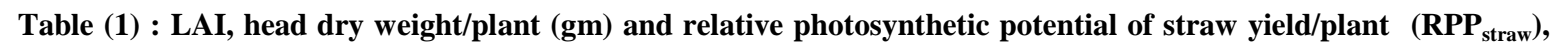
gm/LAI of safflower due to various treatments during 2002/2003 and 2003/2004 seasons.

\begin{tabular}{|c|c|c|c|c|c|c|c|c|c|}
\hline \multirow{2}{*}{ Main effects and interactions } & \multicolumn{3}{|c|}{ LAI } & \multicolumn{3}{|c|}{ Head dry weight/plant (gm) } & \multicolumn{3}{|c|}{$\mathbf{R P P}_{\text {straw }}(\mathrm{gm} / \mathrm{LAI})$} \\
\hline & Season 1 & Season 2 & Comb. & Season 1 & Season 2 & Comb. & Season 1 & Season 2 & Comb. \\
\hline \multicolumn{10}{|l|}{ Cultivar, V } \\
\hline Giza $1, V_{1}$ & 4.02 & 4.00 & 4.01 & $40.30^{\mathrm{a}}$ & $38.28^{\mathrm{a}}$ & $39.29^{\mathrm{a}}$ & $24.18^{\mathrm{b}}$ & $23.86^{\mathrm{b}}$ & $24.02^{\mathrm{b}}$ \\
\hline Demo $112, V_{2}$ & 4.30 & 4.24 & 4.27 & $44.48^{\mathrm{b}}$ & $42.26^{\mathrm{b}}$ & $43.37^{\mathrm{b}}$ & $23.12^{\mathrm{a}}$ & $23.12^{\mathrm{a}}$ & $23.12^{\mathrm{a}}$ \\
\hline F. test & N.S & N.S & N.S & $*$ & $* *$ & $* *$ & $*$ & $*$ & $*$ \\
\hline \multicolumn{10}{|l|}{ Planting pattern, $\mathbf{P}$} \\
\hline $60 \times 20 \mathrm{~cm} \times 1$ plant, 1 side, $P_{1}$ & $2.68^{\mathrm{a}}$ & $2.64^{\mathrm{a}}$ & $2.66^{\mathrm{a}}$ & $46.60^{\mathrm{d}}$ & $43.74^{\mathrm{d}}$ & $45.17^{\mathrm{d}}$ & $33.40^{\mathrm{d}}$ & $33.66^{\mathrm{d}}$ & $33.53^{\mathrm{d}}$ \\
\hline $60 \times 30 \mathrm{~cm} \times 1$ plant, 1 side, $\mathrm{P}_{2}$ & $3.36^{\mathrm{b}}$ & $3.32^{\mathrm{b}}$ & $3.34^{\mathrm{b}}$ & $43.44^{\mathrm{c}}$ & $41.44^{\mathrm{c}}$ & $42.44^{\mathrm{c}}$ & $26.96^{\mathrm{c}}$ & $26.20^{\mathrm{c}}$ & $26.58^{\mathrm{c}}$ \\
\hline $60 \times 20 \mathrm{~cm} \times 2$ plant, 1 side, $P_{3}$ & $4.84^{\mathrm{c}}$ & $4.80^{\mathrm{c}}$ & $4.82^{\mathrm{c}}$ & $40.64^{\mathrm{b}}$ & $39.18^{\mathrm{b}}$ & $39.91^{\mathrm{b}}$ & $19.02^{\mathrm{b}}$ & $19.26^{\mathrm{b}}$ & $19.14^{\mathrm{b}}$ \\
\hline $60 \times 30 \mathrm{~cm} \times 2$ plant, 2 side, $\mathrm{P}_{4}$ & $5.76^{\mathrm{d}}$ & $5.72^{\mathrm{d}}$ & $5.74^{\mathrm{d}}$ & $38.88^{\mathrm{a}}$ & $36.72^{\mathrm{a}}$ & $37.80^{\mathrm{a}}$ & $15.22^{\mathrm{a}}$ & $14.84^{\mathrm{a}}$ & $15.03^{\mathrm{a}}$ \\
\hline F. test & $* *$ & $* *$ & $* *$ & $*$ & $* *$ & $* *$ & $* *$ & $* *$ & $* *$ \\
\hline \multicolumn{10}{|l|}{$\mathrm{N}$ levels, kg N/fed, $\mathbf{N}$ : } \\
\hline Check & $3.36^{\mathrm{a}}$ & $3.28^{\mathrm{a}}$ & $3.32^{\mathrm{a}}$ & $30.21^{\mathrm{a}}$ & $27.73^{\mathrm{a}}$ & $28.97^{\mathrm{a}}$ & $22.55^{\mathrm{a}}$ & $21.83^{\mathrm{a}}$ & $22.19^{\mathrm{a}}$ \\
\hline 40 & $4.30^{\mathrm{b}}$ & $4.28^{\mathrm{b}}$ & $4.29^{\mathrm{b}}$ & $47.67^{\mathrm{b}}$ & $44.65^{\mathrm{b}}$ & $46.16^{\mathrm{b}}$ & $23.40^{\mathrm{b}}$ & $23.60^{\mathrm{b}}$ & $23.50^{\mathrm{b}}$ \\
\hline 80 & $4.82^{c}$ & $4.80^{\mathrm{c}}$ & $4.81^{\mathrm{c}}$ & $49.29^{c}$ & $48.43^{\mathrm{c}}$ & $48.86^{\mathrm{c}}$ & $25.00^{\mathrm{c}}$ & $25.04^{\mathrm{c}}$ & $25.02^{\mathrm{c}}$ \\
\hline F. test & $* *$ & $* *$ & $* *$ & $* *$ & $* *$ & $* *$ & $* *$ & $* *$ & $* *$ \\
\hline \multicolumn{10}{|l|}{ Interactions } \\
\hline $\mathrm{V} \times \mathrm{P}$ & $*$ & N.S & N.S & N.S & $*$ & N.S & $*$ & N.S & N.S \\
\hline $\mathrm{V} \times \mathrm{N}$ & $*$ & $*$ & $*$ & $*$ & $*$ & $*$ & N.S & $*$ & $*$ \\
\hline $\mathrm{P} \times \mathrm{N}$ & * & $*$ & $*$ & * & N.S & $*$ & $*$ & $*$ & $*$ \\
\hline
\end{tabular}

$\mathrm{P}_{1}=35000$ plants/fed $\quad \mathrm{P}_{2}=70000$ plants/fed $\quad \mathrm{P}_{3}=46666$ plants/fed $\quad \mathrm{P}_{4}=93333$ plants $/$ fed

$1 \%, 5 \%$ and NS : refer to the significance level at 1 and $5 \%$ and insignificant differences.

Table (2) : Relative photosynthetic potential of both seed and biological yield/plant, gm/LAI, $\left(\mathbf{R P P}_{\text {seed }}, \mathbf{R P P}_{\text {biol }}\right)$ and migration coefficient (MC)of safflower due to various treatments during $2002 / 2003$ and 2003/2004 seasons.

\begin{tabular}{|c|c|c|c|c|c|c|c|c|c|}
\hline \multirow{2}{*}{ Main effects and interactions } & \multicolumn{3}{|c|}{$\mathbf{R P P}_{\text {seed }}, \mathbf{g m} / \mathbf{L A I}$} & \multicolumn{3}{|c|}{$\mathbf{R P P}_{\text {biol }}, \mathbf{g m} / \mathbf{L A I}$} & \multicolumn{3}{|c|}{ Migration coefficient, MC } \\
\hline & Season 1 & Season 2 & Comb. & Season 1 & Season 2 & Comb. & Season 1 & Season 2 & Comb. \\
\hline \multicolumn{10}{|l|}{ Cultivar, V } \\
\hline$\overline{\text { Giza } 1,} \mathrm{~V}_{1}$ & $5.20 \mathrm{a}$ & $4.72 \mathrm{a}$ & $4.96 \mathrm{a}$ & $29.38 \mathrm{a}$ & $28.58 \mathrm{a}$ & $28.98 \mathrm{a}$ & $0.344 \mathrm{a}$ & $0.338 \mathrm{a}$ & $0.341 \mathrm{a}$ \\
\hline Demo $112, \mathrm{~V}_{2}$ & $7.26 \mathrm{~b}$ & $6.78 \mathrm{~b}$ & $7.02 b$ & $30.38 b$ & $29.90 b$ & $30.14 b$ & $0.358 b$ & $0.348 b$ & $0.353 b$ \\
\hline F. test & $* *$ & $* *$ & $* *$ & & & & & & \\
\hline \multicolumn{10}{|l|}{ Planting pattern, $\mathbf{P}$} \\
\hline $60 \times 20 \mathrm{~cm} \times 1$ plant, 1 side, $P_{1}$ & $10.34 d$ & $9.62 d$ & $9.98 \mathrm{~d}$ & $43.74 d$ & $43.28 \mathrm{~d}$ & $43.51 \mathrm{~d}$ & $0.331 \mathrm{a}$ & $0.313 \mathrm{a}$ & $0.322 \mathrm{a}$ \\
\hline $60 \times 30 \mathrm{~cm} \times 1$ plant, 1 side, $P_{2}$ & $7.31 \mathrm{c}$ & $6.97 \mathrm{c}$ & $7.14 \mathrm{c}$ & $34.27 \mathrm{c}$ & $33.17 \mathrm{c}$ & $33.72 \mathrm{c}$ & $0.343 b$ & $0.345 b$ & $0.344 \mathrm{~b}$ \\
\hline $60 \times 20 \mathrm{~cm} \times 2$ plant, 1 side, $P_{3}$ & $4.29 b$ & $3.73 b$ & $4.01 \mathrm{~b}$ & $23.31 b$ & $22.99 b$ & $23.15 b$ & $0.360 \mathrm{c}$ & $0.350 \mathrm{c}$ & $0.355 \mathrm{c}$ \\
\hline $60 \times 30 \mathrm{~cm} \times 2$ plant, 2 side, $P_{4}$ & $2.98 \mathrm{a}$ & $2.68 \mathrm{a}$ & $2.83 \mathrm{a}$ & $18.20 \mathrm{a}$ & $17.52 \mathrm{a}$ & $17.86 \mathrm{a}$ & $0.370 \mathrm{~d}$ & $0.364 d$ & $0.367 \mathrm{~d}$ \\
\hline F. test & $* *$ & $* *$ & $* *$ & $* *$ & $* *$ & $* *$ & $* *$ & $* *$ & $* *$ \\
\hline \multicolumn{10}{|l|}{ N levels, kg N/fed, $N$ : } \\
\hline Check & $4.66 \mathrm{a}$ & $4.26 \mathrm{a}$ & $4.46 \mathrm{a}$ & $27.21 \mathrm{a}$ & $26.09 \mathrm{a}$ & $26.65 a$ & $0.302 \mathrm{a}$ & $0.300 \mathrm{a}$ & $0.301 \mathrm{a}$ \\
\hline 40 & $6.53 b$ & $6.07 \mathrm{~b}$ & $6.30 \mathrm{~b}$ & $29.93 b$ & $29.67 b$ & $29.80 b$ & $0.363 b$ & $0.353 b$ & $0.358 b$ \\
\hline 80 & $7.50 \mathrm{c}$ & $6.92 \mathrm{c}$ & $7.21 \mathrm{c}$ & $32.50 \mathrm{c}$ & $31.96 \mathrm{c}$ & $32.23 \mathrm{c}$ & $0.388 \mathrm{c}$ & $0.376 \mathrm{c}$ & $0.382 \mathrm{c}$ \\
\hline F. test & $* *$ & $* *$ & $* *$ & $* *$ & $* *$ & $* *$ & $* *$ & $* *$ & $* *$ \\
\hline \multicolumn{10}{|l|}{ Interactions } \\
\hline $\mathrm{V} \times \mathrm{P}$ & $*$ & $*$ & $*$ & $*$ & $*$ & $*$ & N.S & $*$ & ** \\
\hline $\mathrm{V} \times \mathrm{N}$ & $*$ & $*$ & $*$ & $*$ & $* *$ & * & $*$ & N.S & N.S \\
\hline $\mathrm{P} \times \mathrm{N}$ & $*$ & $*$ & $*$ & $* *$ & $*$ & $* *$ & $*$ & $*$ & $*$ \\
\hline
\end{tabular}

Table (3) : Straw, seed and biological (biomass) yield/plant (gm) of safflower due to various treatments during 2002/2003 and 2003/2004 seasons.

\begin{tabular}{|c|c|c|c|c|c|c|c|c|c|}
\hline \multirow{2}{*}{ Main effects and interactions } & \multicolumn{3}{|c|}{ Straw yield/plant (gm) } & \multicolumn{3}{|c|}{ Seed yield/plant (gm) } & \multicolumn{3}{|c|}{ Biomass/plant (gm) } \\
\hline & Season 1 & Season 2 & Comb. & Season 1 & Season 2 & Comb. & Season 1 & Season 2 & Comb. \\
\hline \multicolumn{10}{|l|}{ Cultivar, V } \\
\hline$\overline{\text { Giza } 1,} \mathrm{~V}_{1}$ & $97.18 \mathrm{a}$ & $94.58 \mathrm{a}$ & $95.88 \mathrm{a}$ & $20.42 \mathrm{a}$ & $18.48 \mathrm{a}$ & $19.45 \mathrm{a}$ & $117.60 \mathrm{a}$ & $113.06 \mathrm{a}$ & $115.33 a$ \\
\hline Demo $112, \mathrm{~V}_{2}$ & $99.42 b$ & $98.90 \mathrm{~b}$ & $99.16 b$ & $24.60 b$ & $22.38 b$ & $23.49 b$ & $124.02 b$ & $121.22 \mathrm{~b}$ & $122.65 b$ \\
\hline F. test & $*$ & $* *$ & $* *$ & $*$ & $* *$ & $*$ & $* *$ & $* *$ & $* *$ \\
\hline \multicolumn{10}{|l|}{ Planting pattern, $\mathbf{P}$} \\
\hline $60 \times 20 \mathrm{~cm} \times 1$ plant, 1 side, $P_{1}$ & $112.98 \mathrm{~d}$ & $112.66 \mathrm{~d}$ & $112.82 \mathrm{~d}$ & $27.72 d$ & $25.40 \mathrm{~d}$ & $26.56 \mathrm{~d}$ & $140.70 \mathrm{~d}$ & $138.06 \mathrm{~d}$ & $139.38 \mathrm{~d}$ \\
\hline $60 \times 30 \mathrm{~cm} \times 1$ plant, 1 side, $P_{2}$ & $100.42 \mathrm{c}$ & $96.98 \mathrm{c}$ & $98.70 \mathrm{c}$ & $24.56 \mathrm{c}$ & $23.10 \mathrm{c}$ & $23.83 c$ & $124.98 \mathrm{c}$ & $120.08 \mathrm{c}$ & $122.53 \mathrm{c}$ \\
\hline $60 \times 20 \mathrm{~cm} \times 2$ plant, 1 side, $P_{3}$ & $92.06 \mathrm{~b}$ & $92.44 \mathrm{~b}$ & $92.25 b$ & $20.76 b$ & $17.84 b$ & $19.30 \mathrm{~b}$ & $112.82 b$ & $110.28 b$ & $111.55 b$ \\
\hline $60 \times 30 \mathrm{~cm} \times 2$ plant, 2 side, $\mathrm{P}_{4}$ & $87.74 \mathrm{a}$ & $84.88 \mathrm{a}$ & $86.31 \mathrm{a}$ & $17.00 \mathrm{a}$ & $15.38 \mathrm{a}$ & $16.19 \mathrm{a}$ & $104.74 \mathrm{a}$ & $100.26 \mathrm{a}$ & $102.50 \mathrm{a}$ \\
\hline F. test & $* *$ & & $* *$ & $* *$ & $* *$ & $* *$ & $* *$ & $* *$ & $* *$ \\
\hline \multicolumn{10}{|l|}{ N levels, kg N/fed, $N$ : } \\
\hline Check & $75.96 \mathrm{a}$ & $72.22 \mathrm{a}$ & $74.09 a$ & $12.33 \mathrm{a}$ & $10.89 \mathrm{a}$ & $11.61 \mathrm{a}$ & $88.29 \mathrm{a}$ & $83.20 \mathrm{a}$ & $85.70 \mathrm{a}$ \\
\hline 40 & $100.71 b$ & $101.21 \mathrm{~b}$ & $100.96 b$ & $23.79 b$ & $21.81 b$ & $22.80 b$ & $124.50 \mathrm{~b}$ & $123.02 \mathrm{~b}$ & $123.76 b$ \\
\hline 80 & $118.23 \mathrm{c}$ & $116.79 \mathrm{c}$ & $117.51 \mathrm{c}$ & $31.41 \mathrm{c}$ & $28.59 \mathrm{c}$ & $30.00 \mathrm{c}$ & $149.64 c$ & $145.38 \mathrm{c}$ & $147.51 \mathrm{c}$ \\
\hline F. test & $* *$ & $* *$ & $* *$ & $* *$ & $* *$ & $* *$ & $* *$ & $* *$ & $* *$ \\
\hline \multicolumn{10}{|l|}{ Interactions } \\
\hline $\mathrm{V} \times \mathrm{P}$ & $*$ & $*$ & $*$ & $*$ & N.S & $*$ & $*$ & $*$ & $*$ \\
\hline $\mathrm{V} \times \mathrm{N}$ & $*$ & $*$ & $* *$ & $*$ & $*$ & $*$ & $* *$ & $*$ & $* *$ \\
\hline $\mathrm{P} \times \mathrm{N}$ & $*$ & $* *$ & $* *$ & $*$ & $*$ & $*$ & $* *$ & $* *$ & $* *$ \\
\hline
\end{tabular}




\begin{tabular}{|c|c|c|c|c|c|c|c|c|c|c|c|c|c|c|}
\hline \multicolumn{15}{|c|}{$\begin{array}{l}\text { Table (4) : Straw, seed and biological yield/plant (gm) and their RPP as well as migration coefficient of safflower due to the V } x \\
\text { P interaction (pooled data). }\end{array}$} \\
\hline \multirow{3}{*}{$\begin{array}{l}\text { Planting } \\
\text { pattern, } \mathbf{P}\end{array}$} & \multicolumn{2}{|c|}{$\begin{array}{l}\text { RPP } \\
(\text { straw } \\
\mathbf{L A I})\end{array}$} & \multicolumn{2}{|c|}{\begin{tabular}{|c|}
$\mathbf{R P P}$ \\
$(\mathbf{g m} / \mathbf{L A I})$
\end{tabular}} & \multicolumn{2}{|c|}{\begin{tabular}{c|}
$\mathbf{R P P}$ \\
$(\mathbf{g m} / \mathbf{L A I} \mathbf{A})$
\end{tabular}} & \multicolumn{2}{|c|}{ MC } & \multicolumn{2}{|c|}{\begin{tabular}{|c|} 
Straw yield/plant \\
(gm)
\end{tabular}} & \multicolumn{2}{|c|}{$\begin{array}{l}\text { Seed yield/plant } \\
\text { (gm) }\end{array}$} & \multicolumn{2}{|c|}{$\begin{array}{c}\text { Biological yield/plant } \\
\text { (gm) }\end{array}$} \\
\hline & \multicolumn{14}{|c|}{ Cultivars, $\mathbf{V}$} \\
\hline & $\mathrm{V}_{1}$ & $\mathrm{~V}_{2}$ & $\overline{V_{1}}$ & $\mathrm{~V}_{2}$ & $\mathrm{~V}_{1}$ & $\mathbf{V}_{2}$ & $\overline{V_{1}}$ & $\mathbf{V}_{2}$ & $\overline{V_{1}}$ & $\mathbf{V}_{2}$ & $\overline{V_{1}}$ & $\mathrm{~V}_{2}$ & $\overline{V_{1}}$ & $\mathrm{~V}_{2}$ \\
\hline$\overline{\mathbf{P}_{1}}$ & $\begin{array}{c}\text { B } \\
34.20 \mathrm{~d}\end{array}$ & $\begin{array}{c}\mathrm{A} \\
32.86 \mathrm{~d}\end{array}$ & $\begin{array}{c}\mathrm{A} \\
9.49 \mathrm{~d}\end{array}$ & $\begin{array}{c}\mathrm{B} \\
10.47 \mathrm{~d}\end{array}$ & \begin{tabular}{|c|}
$\mathrm{A}$ \\
$43.13 \mathrm{~d}$
\end{tabular} & \begin{tabular}{|c|}
$\mathrm{B}$ \\
$43.89 \mathrm{~d}$
\end{tabular} & \begin{tabular}{|c|}
$\mathrm{A}$ \\
$0.319 \mathrm{a}$
\end{tabular} & $\begin{array}{c}\text { B } \\
0.325 a\end{array}$ & \begin{tabular}{|c|}
$A$ \\
$110.40 \mathrm{~d}$
\end{tabular} & \begin{tabular}{|c|}
$\mathrm{B}$ \\
$115.24 \mathrm{~d}$
\end{tabular} & $\begin{array}{c}\mathrm{A} \\
24.48 \mathrm{~d}\end{array}$ & $\begin{array}{c}\text { B } \\
28.64 d\end{array}$ & $\begin{array}{c}\mathrm{A} \\
134.00 \mathrm{~d}\end{array}$ & $\begin{array}{c}\mathrm{B} \\
144.76 \mathrm{~d}\end{array}$ \\
\hline $\mathbf{P}_{2}$ & $\begin{array}{c}\mathrm{B} \\
27.18 \mathrm{c}\end{array}$ & \begin{tabular}{|c|}
$\mathrm{A}$ \\
$25.98 \mathrm{c}$ \\
\end{tabular} & $\begin{array}{c}\mathrm{A} \\
6.09 \mathrm{c}\end{array}$ & \begin{tabular}{|c|}
$\mathrm{B}$ \\
$8.19 \mathrm{c}$
\end{tabular} & \begin{tabular}{|c|}
$\mathrm{A}$ \\
$33.23 \mathrm{c}$ \\
\end{tabular} & \begin{tabular}{|c|}
$\mathrm{B}$ \\
$34.21 \mathrm{c}$ \\
\end{tabular} & \begin{tabular}{c|}
$\mathrm{A}$ \\
$0.340 \mathrm{~b}$ \\
\end{tabular} & $\begin{array}{c}\mathrm{B} \\
0.348 \mathrm{~b}\end{array}$ & $\begin{array}{c}\mathrm{A} \\
96.60 \mathrm{c}\end{array}$ & $\begin{array}{c}\mathrm{B} \\
100.80 \mathrm{c}\end{array}$ & $\begin{array}{c}\mathrm{A} \\
21.50 \mathrm{c} \\
\end{array}$ & $\begin{array}{c}\mathrm{B} \\
26.16 \mathrm{c}\end{array}$ & $\begin{array}{c}\mathrm{A} \\
118.00 \mathrm{c}\end{array}$ & $\begin{array}{c}\mathrm{B} \\
127.06 \mathrm{c}\end{array}$ \\
\hline$\overline{\mathbf{P}_{3}}$ & $\begin{array}{c}\mathrm{B} \\
19.40 \mathrm{~b} \\
\end{array}$ & \begin{tabular}{|c|}
$\mathrm{A}$ \\
$18.88 \mathrm{~b}$ \\
\end{tabular} & $\begin{array}{c}\mathrm{A} \\
2.64 \mathrm{~b} \\
\end{array}$ & \begin{tabular}{c|}
$\mathrm{B}$ \\
$5.38 \mathrm{~b}$ \\
\end{tabular} & \begin{tabular}{|c|}
$\mathrm{A}$ \\
$22.30 \mathrm{~b}$ \\
\end{tabular} & \begin{tabular}{|c|}
$\mathrm{B}$ \\
$24.00 \mathrm{~b}$ \\
\end{tabular} & \begin{tabular}{c|}
$\mathrm{A}$ \\
$0.345 \mathrm{c}$ \\
\end{tabular} & $\begin{array}{c}\mathrm{B} \\
0.365 \mathrm{c}\end{array}$ & $\begin{array}{c}\mathrm{A} \\
91.20 \mathrm{~b}\end{array}$ & $\begin{array}{c}\mathrm{B} \\
93.30 \mathrm{~b}\end{array}$ & \begin{tabular}{c|}
$\mathrm{A}$ \\
$17.40 \mathrm{~b}$
\end{tabular} & $\begin{array}{c}\mathrm{B} \\
21.20 \mathrm{~b}\end{array}$ & $\begin{array}{c}\mathrm{A} \\
109.00 \mathrm{~b}\end{array}$ & $\begin{array}{c}\mathrm{B} \\
114.10 \mathrm{~b}\end{array}$ \\
\hline $\mathbf{P}_{4}$ & $\begin{array}{c}\mathrm{B} \\
15.30 \mathrm{a}\end{array}$ & \begin{tabular}{|c|}
$\mathrm{A}$ \\
$14.76 \mathrm{a}$ \\
\end{tabular} & $\begin{array}{c}\mathrm{A} \\
1.62 \mathrm{a}\end{array}$ & $\begin{array}{c}\mathrm{B} \\
4.04 \mathrm{a} \\
\end{array}$ & $\begin{array}{c}\mathrm{A} \\
17.26 \mathrm{a} \\
\end{array}$ & $\begin{array}{c}\mathrm{B} \\
18.46 \mathrm{a}\end{array}$ & $\begin{array}{c}\mathrm{A} \\
0.360 \mathrm{~d}\end{array}$ & $\begin{array}{c}\mathrm{B} \\
0.374 \mathrm{~d}\end{array}$ & $\begin{array}{c}\mathrm{A} \\
85.32 \mathrm{a}\end{array}$ & $\begin{array}{c}\mathrm{B} \\
87.30 \mathrm{a}\end{array}$ & $\begin{array}{c}\mathrm{A} \\
14.42 \mathrm{a} \\
\end{array}$ & $\begin{array}{c}\mathrm{B} \\
17.96 \mathrm{a}\end{array}$ & $\begin{array}{c}\mathrm{A} \\
100.32 \mathrm{a}\end{array}$ & $\begin{array}{c}\mathrm{B} \\
104.68 \mathrm{a}\end{array}$ \\
\hline & dltivar & & & $\mathrm{o} 112 \mathrm{c}$ & ultivar & & & & & & & & & \\
\hline
\end{tabular}

\begin{tabular}{|c|c|c|c|c|c|c|c|c|c|c|c|c|c|c|c|c|}
\hline \multirow{3}{*}{$\begin{array}{c}\mathbf{N} \\
\text { levels } \\
\text { (kg/fed.) }\end{array}$} & \multicolumn{2}{|c|}{ LAI } & \multicolumn{2}{|c|}{\begin{tabular}{|c|} 
Head dry \\
weight/plant \\
(gm)
\end{tabular}} & \multicolumn{2}{|c|}{$\begin{array}{c}\mathbf{R P P}_{\text {straw }} \\
\text { (gm/LAI) }\end{array}$} & \multicolumn{2}{|c|}{$\begin{array}{c}\mathbf{R P P}_{\text {seed }} \\
(\mathbf{g m} / \mathbf{L A I})\end{array}$} & \multicolumn{2}{|c|}{$\begin{array}{l}\mathbf{R P P}_{\text {biol }} \\
\text { (gm/LAI) }\end{array}$} & \multicolumn{2}{|c|}{$\begin{array}{c}\text { Straw } \\
\text { yield/plant (gm) }\end{array}$} & \multicolumn{2}{|c|}{$\begin{array}{c}\text { Seed yield/plant } \\
(\mathrm{gm})\end{array}$} & \multicolumn{2}{|c|}{$\begin{array}{c}\text { Biomass } \\
\text { yield/plant (gm) }\end{array}$} \\
\hline & \multicolumn{16}{|c|}{ Cultivars, V } \\
\hline & $V_{1}$ & $V_{2}$ & $V_{1}$ & $V_{2}$ & $V_{1}$ & $\mathbf{V}_{2}$ & $V_{1}$ & $V_{1}$ & $V_{2}$ & $V_{2}$ & $V_{1}$ & $V_{2}$ & $V_{1}$ & $V_{2}$ & $\mathbf{V}_{1}$ & $V_{2}$ \\
\hline Check & \begin{tabular}{|c|}
$\mathrm{A}$ \\
$3.24 \mathrm{a}$
\end{tabular} & $\begin{array}{c}\mathrm{B} \\
3.40 \mathrm{a}\end{array}$ & \begin{tabular}{|c|}
$\mathrm{A}$ \\
$27.35 \mathrm{a}$
\end{tabular} & \begin{tabular}{|c|}
$\mathrm{B}$ \\
$30.59 \mathrm{a}$
\end{tabular} & $\begin{array}{c}\mathrm{B} \\
22.38 \mathrm{a}\end{array}$ & $\begin{array}{c}\mathrm{A} \\
22.00 \mathrm{a}\end{array}$ & $\begin{array}{c}\mathrm{A} \\
4.00 \mathrm{a}\end{array}$ & \begin{tabular}{|c|}
$\mathrm{B}$ \\
$4.92 \mathrm{a}$
\end{tabular} & \begin{tabular}{|c|}
$\mathrm{A}$ \\
$26.00 \mathrm{a}$
\end{tabular} & \begin{tabular}{|c|}
$\mathrm{B}$ \\
$27.30 \mathrm{a}$
\end{tabular} & $\begin{array}{c}A \\
72.15 \mathrm{a}\end{array}$ & $\begin{array}{c}\mathrm{B} \\
76.06 \mathrm{a}\end{array}$ & $\begin{array}{c}\mathrm{A} \\
10.50 \mathrm{a}\end{array}$ & \begin{tabular}{|c|}
$\mathrm{B}$ \\
$12.72 \mathrm{a}$
\end{tabular} & $\begin{array}{c}\mathrm{A} \\
80.00 \mathrm{a}\end{array}$ & $\begin{array}{c}\mathrm{B} \\
91.40 \mathrm{a}\end{array}$ \\
\hline 40 & $\begin{array}{c}\mathrm{A} \\
4.15 \mathrm{~b}\end{array}$ & $\begin{array}{c}\mathrm{B} \\
4.43 \mathrm{~b}\end{array}$ & $\begin{array}{c}\mathrm{A} \\
44.27 \mathrm{~b}\end{array}$ & \begin{tabular}{|c|}
$\mathrm{B}$ \\
$48.05 \mathrm{~b}$
\end{tabular} & $\begin{array}{c}\mathrm{B} \\
23.86 \mathrm{~b}\end{array}$ & \begin{tabular}{|c|}
$\mathrm{A}$ \\
$23.14 \mathrm{~b}$
\end{tabular} & $\begin{array}{c}\mathrm{A} \\
5.02 \mathrm{~b}\end{array}$ & $\begin{array}{c}\mathrm{B} \\
7.58 \mathrm{~b}\end{array}$ & \begin{tabular}{|c|}
$\mathrm{A}$ \\
$29.00 \mathrm{~b}$
\end{tabular} & $\begin{array}{c}\mathrm{B} \\
30.60 \mathrm{~b}\end{array}$ & $\begin{array}{c}\mathrm{A} \\
98.70 \mathrm{~b}\end{array}$ & $\begin{array}{c}\mathrm{B} \\
103.22 \mathrm{~b}\end{array}$ & $\begin{array}{c}\mathrm{A} \\
21.40 \mathrm{~b}\end{array}$ & $\begin{array}{c}\mathrm{B} \\
24.20 \mathrm{~b}\end{array}$ & $\begin{array}{c}\mathrm{A} \\
119.99 \mathrm{~b}\end{array}$ & $\begin{array}{c}\mathrm{B} \\
127.53 \mathrm{~b}\end{array}$ \\
\hline 80 & $\begin{array}{c}\mathrm{A} \\
4.64 \mathrm{c}\end{array}$ & $\begin{array}{c}\mathrm{B} \\
4.98 \mathrm{c}\end{array}$ & $\begin{array}{c}\text { A } \\
46.25 c\end{array}$ & $\begin{array}{c}\mathrm{B} \\
51.47 \mathrm{c}\end{array}$ & $\begin{array}{c}\mathrm{B} \\
25.82 \mathrm{c}\end{array}$ & $\begin{array}{c}\mathrm{A} \\
24.22 \mathrm{c}\end{array}$ & \begin{tabular}{|c|}
$\mathrm{A}$ \\
$5.86 \mathrm{c}$ \\
\end{tabular} & \begin{tabular}{|c|} 
\\
$8.56 \mathrm{c}$ \\
\end{tabular} & \begin{tabular}{|c|}
$\mathrm{A}$ \\
$31.94 \mathrm{c}$
\end{tabular} & $\begin{array}{c}\mathrm{B} \\
32.52 \mathrm{c}\end{array}$ & $\begin{array}{c}\mathrm{A} \\
116.82 \mathrm{c}\end{array}$ & $\begin{array}{c}\mathrm{B} \\
118.20 \mathrm{c}\end{array}$ & \begin{tabular}{c|c}
$\mathrm{A}$ \\
$26.45 \mathrm{c}$
\end{tabular} & $\begin{array}{c}\mathrm{B} \\
33.55 \mathrm{c}\end{array}$ & $\begin{array}{c}\mathrm{A} \\
146.00 \mathrm{c}\end{array}$ & $\begin{array}{c}\text { B } \\
149.02 \mathrm{c}\end{array}$ \\
\hline$v_{1}-$ Un $_{2}$ & ultivar & & $\mathrm{V}_{2}=$ & Demo & culti & & & & & & & & & & & \\
\hline
\end{tabular}




\begin{tabular}{|c|c|c|c|c|c|c|c|c|c|c|c|c|c|c|c|}
\hline \multicolumn{16}{|c|}{$\begin{array}{l}\text { Table (6-a) : LAI, head dry weight/plant (gm), } \mathbf{R P P}_{\text {straw }}, \mathbf{R P P}_{\text {seed }} \text { and } \mathrm{RPP}_{\text {boil }}(\mathrm{gm} / \mathrm{LAI}) \text { of safflower due to the } \\
\text { P x N interaction (combined data). }\end{array}$} \\
\hline \multirow{3}{*}{$\begin{array}{l}\text { Planting } \\
\text { pattern, } P\end{array}$} & \multicolumn{2}{|c|}{ LAI } & \multicolumn{3}{|c|}{$\begin{array}{c}\text { Head dry } \\
\text { weight/plant (gm) }\end{array}$} & \multicolumn{3}{|c|}{$\mathbf{R P P}_{\text {straw }}(\mathrm{gm} / \mathrm{LAI})$} & \multicolumn{2}{|c|}{$\mathbf{R P P}_{\text {seed }}(\mathrm{gm} / \mathrm{LAI})$} & \multicolumn{5}{|c|}{$\mathbf{R P P}_{\text {biol }}(\mathrm{gm} / \mathrm{LAI})$} \\
\hline & \multicolumn{15}{|c|}{ Nitrogen fertilization levels (kg N/fad), N: } \\
\hline & Check & 40 & 80 & Check & 40 & 80 & Check & 40 & 80 & Check & 40 & 80 & Check & 40 & 80 \\
\hline$\overline{\mathbf{P}_{1}}$ & $\begin{array}{c}\mathrm{A} \\
1.87 \mathrm{a}\end{array}$ & $\begin{array}{c}\mathrm{B} \\
2.84 \mathrm{a}\end{array}$ & $\begin{array}{c}\mathrm{C} \\
3.27 \mathrm{a}\end{array}$ & $\begin{array}{c}\mathrm{A} \\
33.23 \mathrm{~d}\end{array}$ & $\begin{array}{c}\mathrm{B} \\
50.64 \mathrm{~d}\end{array}$ & $\begin{array}{c}\mathrm{C} \\
51.64 \mathrm{~d}\end{array}$ & $\begin{array}{c}\mathrm{A} \\
32.50 \mathrm{~d}\end{array}$ & $\begin{array}{c}\mathrm{B} \\
35.20 \mathrm{~d}\end{array}$ & \begin{tabular}{c|c|}
$\mathrm{C}$ \\
$\mathrm{d}$ & $34.89 \mathrm{~d}$
\end{tabular} & $\begin{array}{c}\mathrm{A} \\
8.11 \mathrm{~d}\end{array}$ & \begin{tabular}{|c|}
$B$ \\
$10.35 d$
\end{tabular} & $\begin{array}{c}\mathrm{C} \\
11.48 \mathrm{~d}\end{array}$ & \begin{tabular}{|c|}
$\mathrm{A}$ \\
$37.30 \mathrm{~d}$
\end{tabular} & $\begin{array}{c}\mathrm{B} \\
46.00 \mathrm{~d}\end{array}$ & $\begin{array}{c}\mathrm{C} \\
47.23 \mathrm{~d}\end{array}$ \\
\hline $\mathbf{P}_{2}$ & $\begin{array}{c}\mathrm{A} \\
2.67 \mathrm{~b} \\
\end{array}$ & $\begin{array}{c}\mathrm{B} \\
3.24 \mathrm{~b} \\
\end{array}$ & $\begin{array}{c}\mathrm{C} \\
4.11 \mathrm{~b}\end{array}$ & $\begin{array}{c}\mathrm{A} \\
30.20 \mathrm{c}\end{array}$ & $\begin{array}{c}\mathrm{B} \\
47.82 \mathrm{c} \\
\end{array}$ & $\begin{array}{c}\mathrm{C} \\
49.30 \mathrm{c} \\
\end{array}$ & $\begin{array}{c}\mathrm{A} \\
24.20 \mathrm{c}\end{array}$ & $\begin{array}{c}\mathrm{B} \\
26.60 \mathrm{c}\end{array}$ & \begin{tabular}{c|c|}
$\mathrm{C}$ \\
$\mathrm{c}$ & $28.94 \mathrm{c}$ \\
\end{tabular} & $\begin{array}{c}\mathrm{A} \\
4.52 \mathrm{c} \\
\end{array}$ & $\begin{array}{c}\mathrm{B} \\
8.05 \mathrm{c} \\
\end{array}$ & $\begin{array}{c}\mathrm{C} \\
8.85 \mathrm{c}\end{array}$ & $\begin{array}{c}\mathrm{A} \\
32.00 \mathrm{c}\end{array}$ & $\begin{array}{c}\mathrm{B} \\
33.00 \mathrm{c}\end{array}$ & $\begin{array}{c}\mathrm{C} \\
36.16 \mathrm{c}\end{array}$ \\
\hline $\mathbf{P}_{3}$ & $\begin{array}{c}\mathrm{A} \\
3.97 \mathrm{c}\end{array}$ & $\begin{array}{c}\mathrm{B} \\
5.04 \mathrm{c}\end{array}$ & $\begin{array}{c}\mathrm{C} \\
5.45 \mathrm{c}\end{array}$ & $\begin{array}{c}\mathrm{A} \\
27.24 \mathrm{~b}\end{array}$ & $\begin{array}{c}\mathrm{B} \\
44.50 \mathrm{~b}\end{array}$ & $\begin{array}{c}\mathrm{C} \\
47.99 \mathrm{~b}\end{array}$ & $\begin{array}{c}\mathrm{A} \\
17.50 \mathrm{~b}\end{array}$ & \begin{tabular}{|c|}
$\mathrm{B}$ \\
$19.20 \mathrm{~b}$
\end{tabular} & \begin{tabular}{|c|c|}
$\mathrm{C}$ \\
$\mathrm{b}$ & $20.72 \mathrm{~b}$ \\
\end{tabular} & $\begin{array}{c}\mathrm{A} \\
3.20 \mathrm{~b}\end{array}$ & $\begin{array}{c}\mathrm{B} \\
3.90 \mathrm{~b}\end{array}$ & \begin{tabular}{|c|}
$\mathrm{C}$ \\
$4.93 \mathrm{~b}$
\end{tabular} & \begin{tabular}{|c|}
$\mathrm{A}$ \\
$21.10 \mathrm{~b}$
\end{tabular} & $\begin{array}{c}\mathrm{B} \\
23.20 \mathrm{~b}\end{array}$ & $\begin{array}{c}\mathrm{C} \\
25.15 \mathrm{~b}\end{array}$ \\
\hline $\mathbf{P}_{4}$ & $\begin{array}{c}\mathrm{A} \\
4.77 \mathrm{~d}\end{array}$ & $\begin{array}{c}\mathrm{B} \\
6.04 \mathrm{~d}\end{array}$ & $\begin{array}{c}\mathrm{C} \\
6.41 \mathrm{~d}\end{array}$ & $\begin{array}{c}\mathrm{A} \\
25.21 \mathrm{a}\end{array}$ & $\begin{array}{c}\mathrm{B} \\
41.68 \mathrm{a}\end{array}$ & $\begin{array}{c}\mathrm{C} \\
46.51 \mathrm{a}\end{array}$ & $\begin{array}{c}\mathrm{A} \\
14.56 \mathrm{a}\end{array}$ & $\begin{array}{c}\mathrm{B} \\
15.00 \mathrm{a}\end{array}$ & \begin{tabular}{|c|c|}
$\mathrm{C}$ \\
$\mathrm{a}$ & $15.33 \mathrm{a}$ \\
\end{tabular} & $\begin{array}{c}\mathrm{A} \\
2.01 \mathrm{a}\end{array}$ & $\begin{array}{c}\mathrm{B} \\
2.90 \mathrm{a}\end{array}$ & $\begin{array}{c}\mathrm{C} \\
3.58 \mathrm{a}\end{array}$ & $\begin{array}{c}\mathrm{A} \\
16.20 \mathrm{a}\end{array}$ & $\begin{array}{c}\mathrm{B} \\
17.00 \mathrm{a}\end{array}$ & $\begin{array}{c}\mathrm{C} \\
20.38 \mathrm{a}\end{array}$ \\
\hline
\end{tabular}

Table (6-b) : Migration coefficient (MC) and the final safflower yield/plant from straw, seed and biomass due to the $\mathbf{N}-\mathbf{P}$ interaction recorded from the combined data.

\begin{tabular}{|c|c|c|c|c|c|c|c|c|c|c|c|c|}
\hline \multirow{3}{*}{$\begin{array}{c}\text { Planting } \\
\text { pattern, } \\
\mathbf{P}\end{array}$} & \multicolumn{3}{|c|}{$\begin{array}{c}\text { Migration coefficient } \\
\text { (MC) }\end{array}$} & \multicolumn{3}{|c|}{$\begin{array}{c}\text { Straw yield/plant } \\
\text { (gm) }\end{array}$} & \multicolumn{2}{|c|}{$\begin{array}{c}\text { Seed yield /plant } \\
\text { (gm) }\end{array}$} & \multicolumn{4}{|c|}{ biomass output /plant (gm) } \\
\hline & \multicolumn{12}{|c|}{ Nitrogen fertilization levels (kg N/fad), $\mathrm{N}$ : } \\
\hline & Check & 40 & 80 & Check & 40 & 80 & Check & 40 & 80 & Check & 40 & 80 \\
\hline $\mathbf{P}_{1}$ & $\begin{array}{c}\mathrm{A} \\
0.290 \mathrm{a}\end{array}$ & $\begin{array}{c}\mathrm{B} \\
0.322 \mathrm{a}\end{array}$ & $\begin{array}{c}\mathrm{C} \\
0.354 \mathrm{a}\end{array}$ & $\begin{array}{c}\mathrm{A} \\
78.00 \mathrm{~d}\end{array}$ & $\begin{array}{c}\text { B } \\
115.00 d\end{array}$ & $\begin{array}{c}C \mathrm{C} \\
145.46 \mathrm{~d}\end{array}$ & $\begin{array}{c}\mathrm{A} \\
15.40 \mathrm{~d}\end{array}$ & \begin{tabular}{|c|}
$\mathrm{B}$ \\
$26.85 \mathrm{~d}$
\end{tabular} & $\begin{array}{c}\mathrm{C} \\
27.43 \mathrm{~d}\end{array}$ & $\begin{array}{c}\mathrm{A} \\
102.80 \mathrm{~d}\end{array}$ & $\begin{array}{c}\text { B } \\
141.04 d\end{array}$ & $\begin{array}{c}\mathrm{C} \\
174.30 \mathrm{~d}\end{array}$ \\
\hline $\mathbf{P}_{2}$ & $\begin{array}{c}\mathrm{A} \\
0.298 \mathrm{~b} \\
\end{array}$ & $\begin{array}{c}\mathrm{B} \\
0.350 \mathrm{~b} \\
\end{array}$ & $\begin{array}{c}\mathrm{C} \\
0.384 \mathrm{~b} \\
\end{array}$ & $\begin{array}{c}\mathrm{A} \\
75.00 \mathrm{c} \\
\end{array}$ & $\begin{array}{c}\mathrm{B} \\
102.00 \mathrm{c}\end{array}$ & $\begin{array}{c}\mathrm{C} \\
119.10 \mathrm{c} \\
\end{array}$ & $\begin{array}{c}\mathrm{A} \\
13.32 \mathrm{c} \\
\end{array}$ & $\begin{array}{c}\mathrm{B} \\
25.75 \mathrm{c} \\
\end{array}$ & \begin{tabular}{|c|}
$\mathrm{C}$ \\
$32.42 \mathrm{c}$ \\
\end{tabular} & $\begin{array}{c}\mathrm{A} \\
90.00 \mathrm{c} \\
\end{array}$ & $\begin{array}{c}\mathrm{B} \\
130.00 \mathrm{c}\end{array}$ & $\begin{array}{c}\mathrm{C} \\
147.59 \mathrm{c}\end{array}$ \\
\hline$\overline{\mathbf{P}_{3}}$ & $\begin{array}{c}\mathrm{A} \\
0.306 \mathrm{c} \\
\end{array}$ & $\begin{array}{c}\mathrm{B} \\
0.370 \mathrm{c} \\
\end{array}$ & $\begin{array}{c}\mathrm{C} \\
0.389 \mathrm{c} \\
\end{array}$ & $\begin{array}{c}\mathrm{A} \\
73.10 \mathrm{~b} \\
\end{array}$ & $\begin{array}{c}\text { B } \\
98.00 b \\
\end{array}$ & $\begin{array}{c}\mathrm{C} \\
105.65 \mathrm{~b} \\
\end{array}$ & $\begin{array}{c}\mathrm{A} \\
10.30 \mathrm{~b} \\
\end{array}$ & \begin{tabular}{|c|} 
B \\
$20.78 b$ \\
\end{tabular} & \begin{tabular}{|c|}
$\mathrm{C}$ \\
$26.82 \mathrm{~b}$ \\
\end{tabular} & $\begin{array}{c}\mathrm{A} \\
80.00 \mathrm{~b} \\
\end{array}$ & $\begin{array}{c}\mathrm{B} \\
120.00 \mathrm{~b}\end{array}$ & $\begin{array}{c}\mathrm{C} \\
134.65 \mathrm{~b} \\
\end{array}$ \\
\hline $\mathbf{P}_{4}$ & \begin{tabular}{|c|}
$\mathrm{A}$ \\
$0.310 \mathrm{~d}$
\end{tabular} & $\begin{array}{c}\text { B } \\
0.390 \mathrm{~d}\end{array}$ & $\begin{array}{c}\mathrm{C} \\
0.401 \mathrm{~d}\end{array}$ & $\begin{array}{c}\mathrm{A} \\
70.26 \mathrm{a}\end{array}$ & $\begin{array}{c}\text { B } \\
88.84 a\end{array}$ & $\begin{array}{c}\mathrm{C} \\
99.83 \mathrm{a}\end{array}$ & $\begin{array}{c}\mathrm{A} \\
7.42 \mathrm{a}\end{array}$ & \begin{tabular}{|c|}
$\mathrm{B}$ \\
$17.82 \mathrm{a}$
\end{tabular} & \begin{tabular}{|c|}
$\mathrm{C}$ \\
$23.33 \mathrm{a}$
\end{tabular} & $\begin{array}{c}\mathrm{A} \\
70.00 \mathrm{a}\end{array}$ & $\begin{array}{c}\mathrm{B} \\
104.00 \mathrm{a}\end{array}$ & $\begin{array}{c}\mathrm{C} \\
133.50 \mathrm{a}\end{array}$ \\
\hline
\end{tabular}


from $\mathrm{RPP}_{\text {seed }}, \mathrm{RPP}_{\text {biol }}$ and the final yield/plant from straw, seed and biomass. On the other hand, the dense plant distribution pattern of 93333 plant/fed. gave a high value from MC when the Demo 112 cultivar was considered (Table 4).

In addition, the safflower plants of Giza $1 \mathrm{cv}$ fertilized with $80 \mathrm{~kg} \mathrm{~N}$ level gave considerable increase in $\mathrm{RPP}_{\text {straw }}$. Besides, the plants of Demo $112 \mathrm{cv}$ received $80 \mathrm{~kg} \mathrm{~N}$ level had pronounced excess in each of : LAI, head dry weight, $\mathrm{RPP}_{\text {seed }}$, $\mathrm{RPP}_{\text {biol }}$ and the final yield /plant from straw seed and biomass.

Moreover, the plants of the dense planting pattern of 93333 plant/fed. receiving the $80 \mathrm{~kg} \mathrm{~N}$ level had the best results as for LAI and MC. Likewise, the plant distribution pattern of 35000 plant/fed. fertilized with $80 \mathrm{~kg} \mathrm{~N}$ dose gave the best mean averages respecting : head dry weight, the final yield/plant from straw, seed and biomass and their relative photosynthetic potentials (Tables 6a and $6 \mathrm{~b}$ ). The results of the interactions recorded between the three factors tested allude to the beneficial additional effects of their treatments to exploit the available growth resources to the best which reflected strongly on improving the dry matter accumulation respects and in turn the final yields per plant.

\section{Conclusion}

It can be concluded from the findings of this paper that, applying the even distribution pattern of proper plant stand (35000 plant/fed. for the final yields/plant and their RPP or 93333 plant/fed. for LAI and MC) and using the $80 \mathrm{~kg} \mathrm{~N}$ level/fed. is recommended treatments for raising the partitioning and migration of more synthythates to economic yield/plant for both Giza 1 and Demo 112 cvs, being more preferable in the case of the later cultivar of greater ability to utilize the photosynthates for the better, especially in newly reclaimed sandy soil of low fertility as found in Khattara Project Farm, Zagazig, Sharkia Governorate, Egypt.

Thus, it is necessary to consider the effect of new cultural practices on photosynthate partitioning parameters and yield of high yielding safflower cultivars if the maximum advantage is to be obtained.

\section{REFERENCES}

Afifi M.M. (1991). Effect of mineral fertilization and sowing dates on growth and yield of safflower plants (Carthamus tinctorius L.)
M.Sc. Thesis, Fac. of Agric., Ain-Shams Univ., Egypt.

Badawi M.A., El-Moursy S.A. and Leilah A.A. (1996). Effect of sowing dates and nitrogen fertilization on growth, yield and its components of safflower (Carthamus tinctorius L.). J. Agric. Sci., Mansoura Univ., Vol. 21 (12) : 4275-4290.

Das M.N. and Giri N.C. (1986). Design and Analysis of Experiments. $2^{\text {nd }}$ Ed., John Wiley and Sons. Inc., New-York, USA.

Duncan D.B. (1955). Multiple Range and Multiple F. tests. Biometrics, Vol. $11: 1-42$.

El-Hariri D.M. and Ahmed M.A. (1993). Response of safflower yield to plant density and nitrogen level. Annals of Agric. Sci., Moshtohor, Vol. 31(2) : 729-738.

El-Wakil A.M. and Darweesh Z.H. (1993). Preliminary evaluation of some exotic and Egyptian safflower genotypes. Egypt. J. Appl. Sci., Vol. 8(3) : 437-447.

Kamel M.S., Shabana R., Mahmoud E.A. and Keshtah M.M. (1982). Association between growth attributes of safflower (Carthamus tinctorious L.) and yield of seed and oil under different production practices. Zeitschrift fur Acker Und Phlan Zenbau., Vol. 151(3):169175.

Khalil A.A.M. (1988). Effect of plant density and nitrogen fertilization on safflower. M.Sc. Thesis, Fac. of Agric., Zagazig Univ., Egypt.

McGraw R.L. (1977). Yield dynamics of florunner peanut (Arachis hypogaea, L.) M.Sc. Thesis, Florida Univ.

Nawar F.R.R. (2002). Effect of plant spacing and nitrogen fertilizer on growth and yield of safflower in calcareous soil. J. Adv. Agric. Res., Vol. 7(4) : 853-861.

Refaat A.M., El-Gamal E.A. and Ahmed S.S. (1994). Physiological and chemical studies on some safflower cultivars. Egypt. J. Hort., Vol. 21(1) : 1-13.

Shweikh Ommamh A.M. (1988). Agronomic studies on safflower (Carthamus tinctorius L.). M.Sc. Thesis, Fac. of Agric., Zagazig Univ., Egypt.

Vidovic J. and Pokorny V. (1971). The effect of different sowing densities and nutrient levels on leaf area index. Production and distribution of dry matter in maize. Piologia. Planta., Vol. $15: 374-382$. 


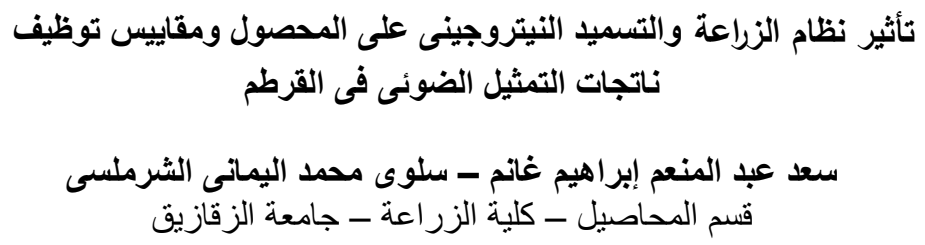

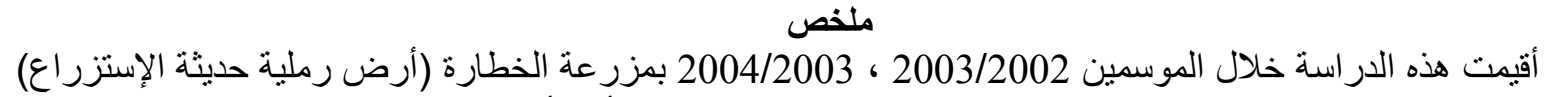

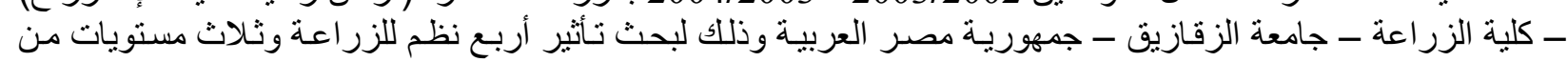

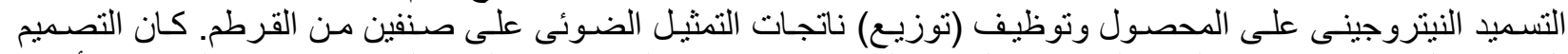

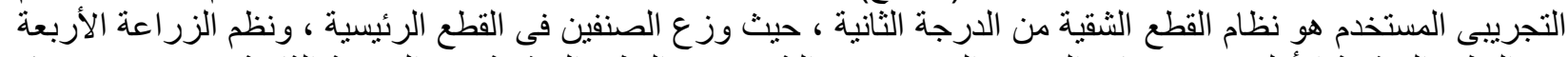

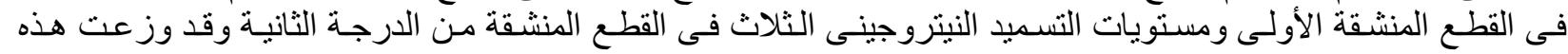

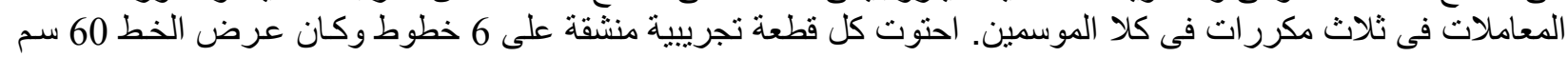

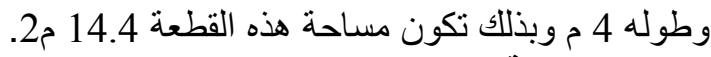

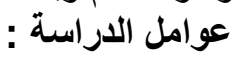
كانت عو امل الدراسة كما يلى : الاسلى : أ أل الأصناف :

$$
\begin{aligned}
& \text { 1 - جيزة } 1 \text { - صنف محلى - }
\end{aligned}
$$

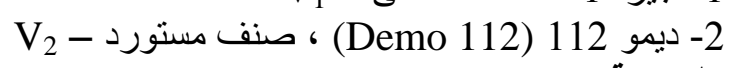

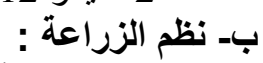

تم استخدام أربع نظم للزر اعة :

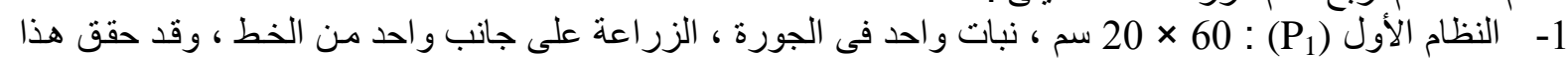
النظام كثافة نباتية (P) : 35000 نبات/فدان.

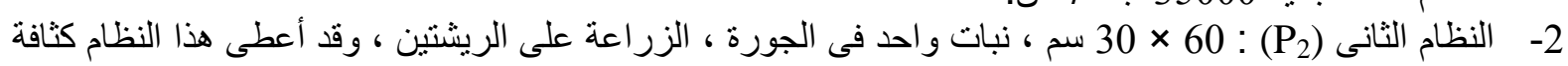
نباتية 46666 نبات/فدان. 3- النظام الثالث (P) : 60 × 600 × 20 سم ، نباتين فى الجورة ، الزر اعة على جانب واحد من الخط، وقد نتج عن هذا

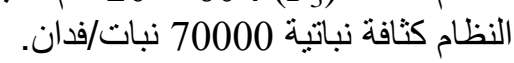

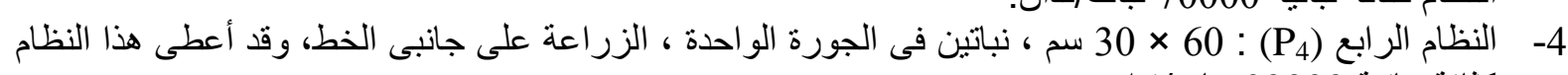
كثافة نباتية

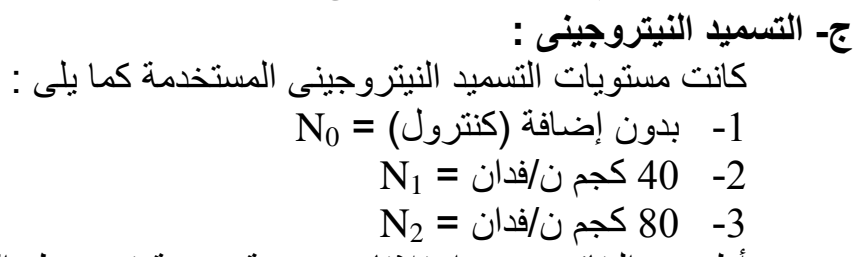

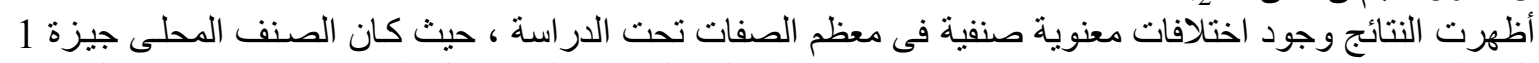

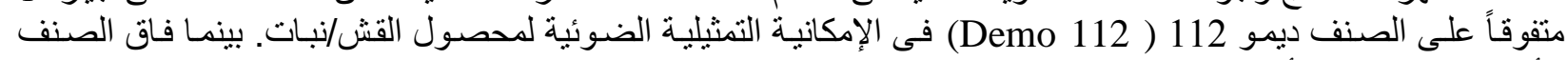

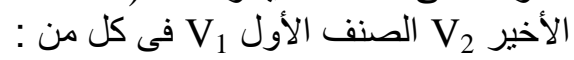

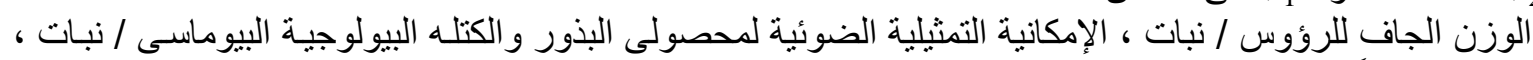

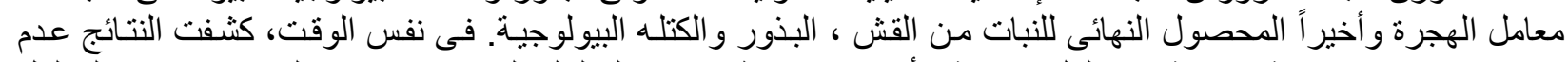

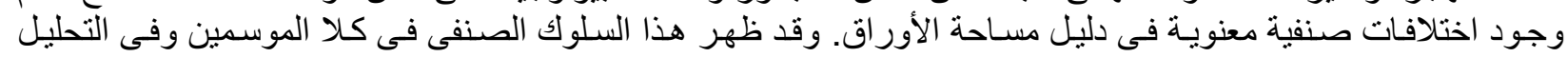

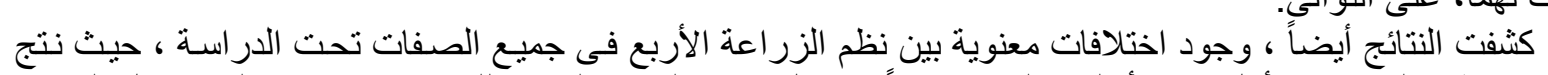

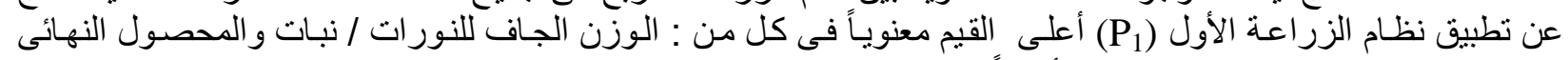

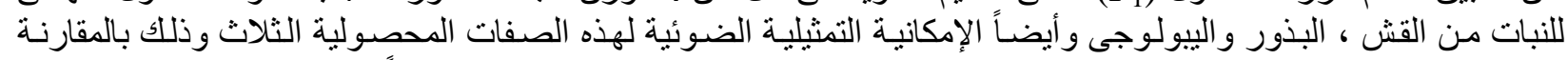

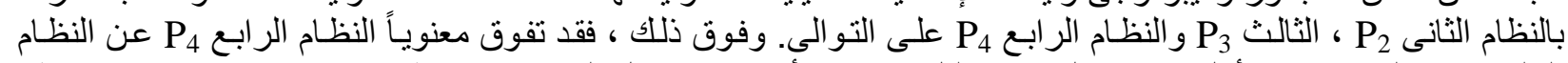

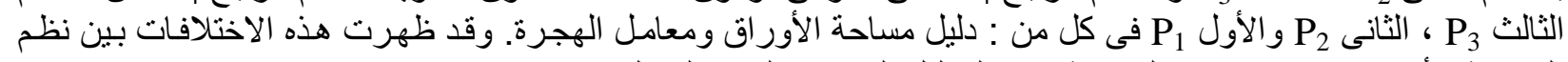

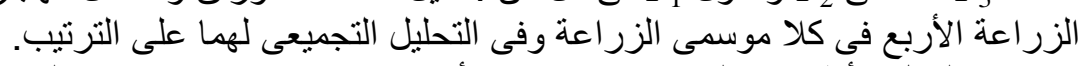

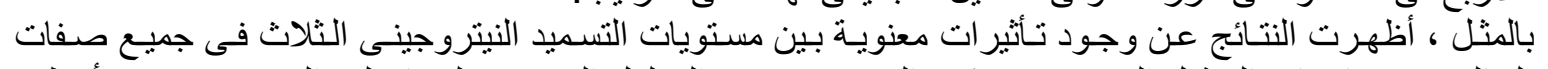

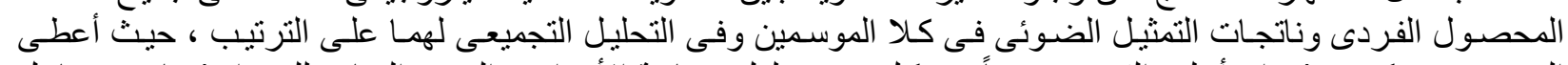

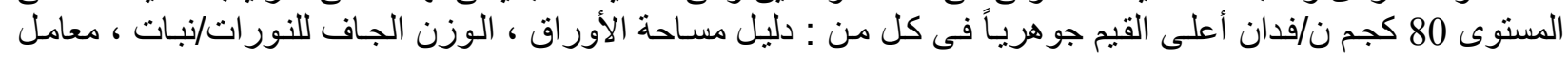




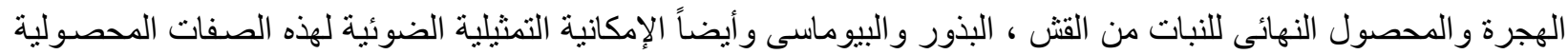

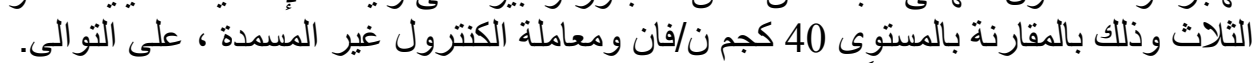

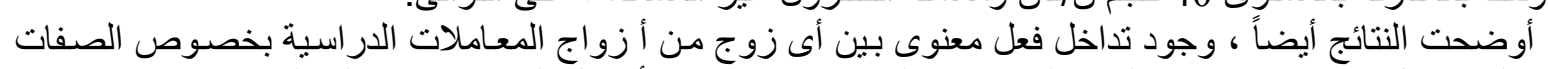

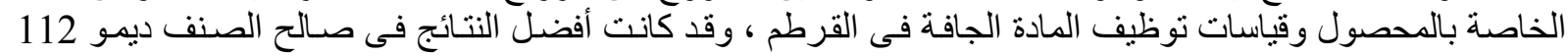

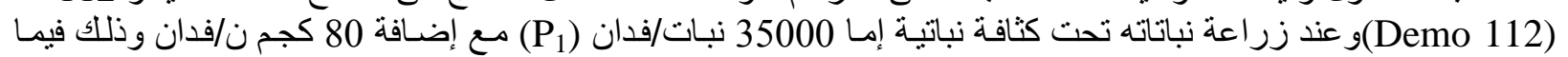

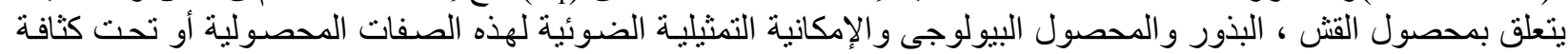

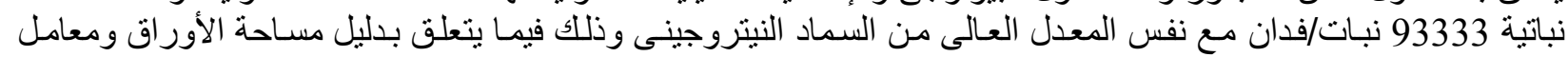

وباختصار ، أظهرت نتائج هذا البحث أن الصنف المستورد 112 Demo ذات كفاءة تمثيلية عالية وذات قدرة فائقة

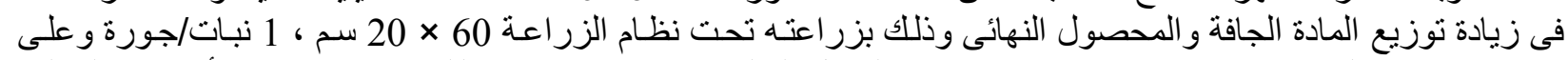

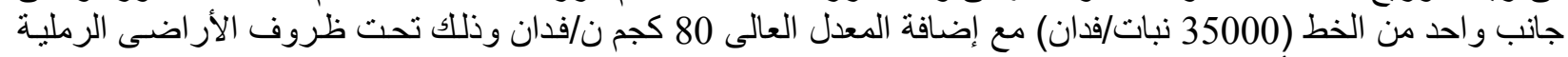

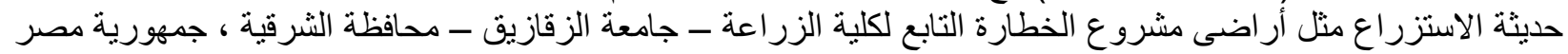

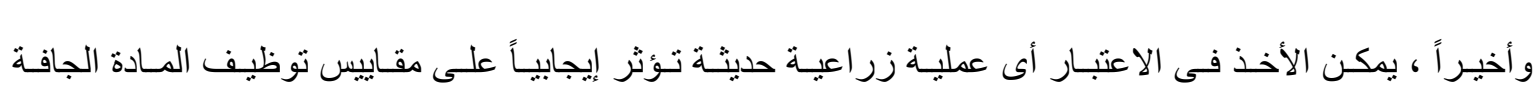

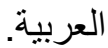

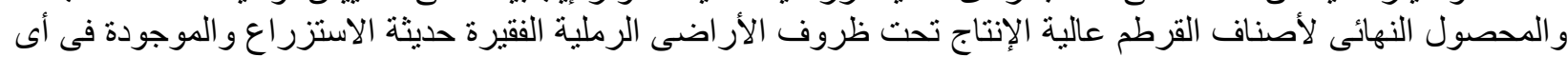
موقع فى الأراضى المصرية.

المجلة العلمية لكلية الزراعة - جامعة القاهرة - المجلد (58) العدد الأول (يناير 2007):1-10 . 